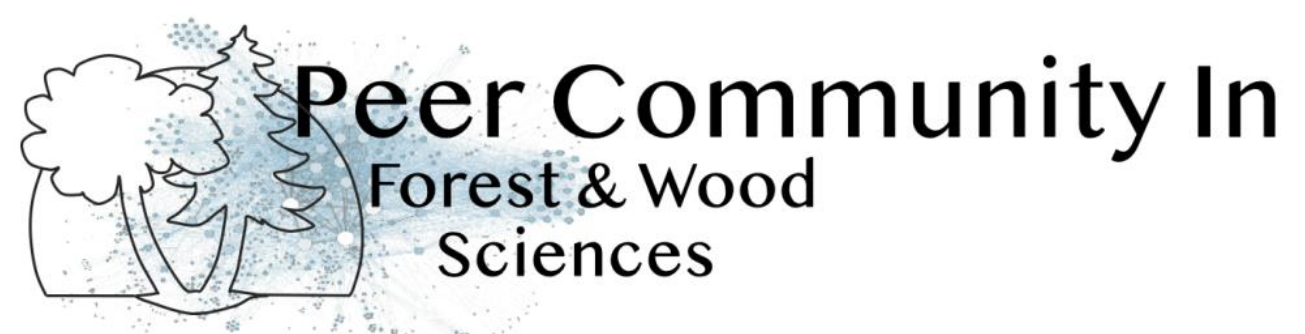

\title{
Black poplar (Populus nigra L.) seed physiology: an important issue for the in situ conservation of this riparian species
}

Erwin Dreyer based on reviews by Eduardo Notivol, Tomasz A.

Pawlowski and 1 anonymous reviewer

\section{A recommendation of:}

Variability in seeds' physicochemical characteristics, germination and seedling growth within and between two French Populus nigra L. populations

Marlène Lefebvre, Marc Villar, Nathalie Boizot, Armelle Delile, Benjamin Dimouro,

\section{Open Access}

Anne-Marie Lomelech, Caroline Teyssier(2020), arXiv, 2008.05744, ver. 3 peer-

reviewed and recommended by Peer Community in Forest \& Wood

Scienceshttps://arxiv.org/abs/2008.05744

Published: 15 March 2021

Copyright: This work is licensed under the Creative Commons

Attribution-NoDerivatives 4.0 International License. To view a copy of this license, visit http://creativecommons.org/licen ses/by-nd/4.0/
Submitted: 14 August 2020, Recommended: 15 March 2021

\section{Recommendation}

Seed physiology, which was a little forgotten in forest ecology since several decades, needs be revitalized as a research area given the many open questions about population dynamics and demography in rapidly changing environments (and not only for endangered species).

Indeed, seed physiology was long mobilized mainly to optimize seed conservation and germination for the production of plant material in a range of tree species used for afforestation/plantation. In the case of back poplar (which by the way is the male genitor of the multiple hybrid Populus $x$ euramerica poplar cultivars), the focus is rather on the conservation ecology of this riparian species, and mainly on in situ conservation (Lefèvre et al, 1998). Indeed, the protection of populations of Populus nigra $L$ requires an improved understanding of the ecology of this species with a focus on reproduction. Indeed, black poplar seeds need to be rapidly disseminated, to germinate as soon as the conditions are favourable (with rather small time windows) and establish seedlings with access to water in the rather harsh environment of mobile and sandy river banks submitted to alternating periods of flooding and of severe water deficits during low river flows in summer (Imbert and Lefèvre, 2003; Corenblit et al, 2014; Tinschert et al, 2020).

This process is therefore central to the propagation/maintenance of these populations that are threatened by the destruction of river banks and by introgression by either genes from the widespread "Italica" cultivar of black poplar, of from other poplar species leading to a variety of natural hybrids (Smulders et al, 2008). 
Many questions remain open about seeds of black poplar (Michalak et al, 2015). One of the most intriguing one is to what extent seed properties and physiology differ within and among local populations from different river catchments. This question was addressed in this preprint by Lefebvre et al. (2021) that provides a very detailed and comparative analysis of two populations from central and southern France, each represented by 10 half sib families (i.e., seed collected separately from 10 adult individuals after open pollination).

Investigated properties were mainly seed biomass, anatomy, germination rate, root growth, lipid and sugar contents, protein content (with identification of some major protein families).

The within populations variability was indeed quite large, but nevertheless there were significant differences between the two populations in several traits, like seed weight, lipid content, and starch content. Storage proteins differed among families, but only slightly between the two populations. However, the main conclusion was that intrinsic qualities of the seeds were not critical for early stage establishment in the two populations, despite some significant differences in mean seed biomass, in lipid and in soluble sugars contents.

The preprint nicely analyses these differences, brings a large set of new observations about the seed physiology of Populus nigra. The referees found the data produced during this research quite important and original. This is why, despite the fact that the number of tested groups of populations remains rather small and the link with seedling establishment remains rather weak, this study is an important contribution to conservation ecology. This research (and that of many other groups) needs be further developed with an emphasis on inter and intra population variation and on demogenetics of forest tree species.

\section{References}

Corenblit D., Steiger J., González, E et al. (2014), The biogeomorphological life cycle of poplars during the fluvial biogeomorphological succession: a special focus on Populus nigra L.. Earth Surf. Process. Landforms, 39: 546-563. doi: https://doi.org/10.1002/esp.3515

Imbert E. and Lefèvre F. (2003) Dispersal and geneflow of Populus nigra (Salicaceae) along a dynamic river system. Journal of Ecology 91: 447-456. doi: https://doi.org/10.1046/j.13652745.2003.00772.x

Lefebvre M., Villar M., Boizot N., Delile A., Dimouro B., Lomelech A.-M. and Teyssier, C. (2021) Variability in seeds' physicochemical characteristics, germination and seedling growth within and between two French Populus nigra populations. arXiv, 2008.05744, ver 3 peer-reviewed and recommended by Peer community in Forest and Wood Sciences. https://arxiv.org/abs/2008.05744

Lefèvre F., Légionnet A., de Vries S. and Turok J. (1998) Strategies for the conservation of a pioneer tree species, Populus nigra L., in Europe. Genetics, Selection, Evolution 30, S181-196. doi: https://doi.org/10.1186/1297-9686-30-S1-S181

Michalak M., Plitta B.P., Tylkowski T. et al. (2015) Desiccation tolerance and cryopreservation of seeds of black poplar (Populus nigra L.), a disappearing tree species in Europe. European Journal of Forest Research 134, 53-60. doi: https://doi.org/10.1007/s10342-014-0832-4

Smulder M.J.M., Beringen R., Volosyanchuk R. et al. (2008) Natural hybridisation between Populus nigra L. and P. $x$ canadensis Moench. Hybrid offspring competes for niches along the Rhine river in the Netherlands. Tree Genetics \& Genomes 4, 663-675. doi: https://doi.org/10.1007/s11295-0080141-5

Tinschert E., Egger G., Wendelgass J. et al. (2020) Alternate reproductive strategies of Populus nigra influence diversity, structure and successional processes within riparian woodlands along the Allier River, France. Journal of Hydro-environment research 30, 100-108.

doi: https://doi.org/10.1016/j.jher.2020.03.004 


\section{Revision round \#2}

\section{1-03-04}

\section{Decision round \#2}

\section{Dear authors}

I have gone through your answers and the new version of the text. You have done an excellent job in revising the manuscript. A few minor typos remain, but they do not justify a new round of revisions. Please correct those typos (listed below), do a last check and post a final version of the manuscript formated according to the instructions given below. Then, I would be very pleased to post my recommendation text on the PCI Forest \& Wood Sciences website.

Thanks in advance

With best regards

\section{Erwin Dreyer}

\section{Last changes to be done}

Here is a list of points of attention for the preprint by Teyssier et al:

1. The link to the original paper is fine on arxiv; I was however unable to find the DOI of the paper, and could only access the text as a pdf document; is this a usual feature for arXiv?

2. The links to the data verse needs be stabilized; I was able to access the data set from the new link you provided, but not from the one reported in the MS;

3. The tables attached to the preprint as "supplementary" material should be referred to as annexes (as they are fully integrated in the main text; supplementary material would be floating on the website with poor connections to the body of the preprint;

4. A series of very minor typos as found in the pdf downloaded from the arXiv site:

a. Please link authors and affiliations on the main page;

b. Please provide also the emails of all co-authors.

C. Abstract: 19.77 should be 19.8

d. In the introduction, there is a change infont size in the § "P. nigra usually forms..."

e. "two orders of magnitude" would mean a factor 100 if I am right... here it is a factor 2;

f. There is an incomplete sentence: "only current-year seeds can germinate and no seed bank." You mean: no seedbank can establish?

g. Light microscopy: the aim of the capture of images is not indicated. Is it purely illustrative of was there some further aim?

h. Change "supplementary table" to "annex" at all occurrences;

i. For lipids, what is meant by "witness" (sun oil)"? is this a control (sorry for sounding naïve);

j. "a graduated ruler"; 


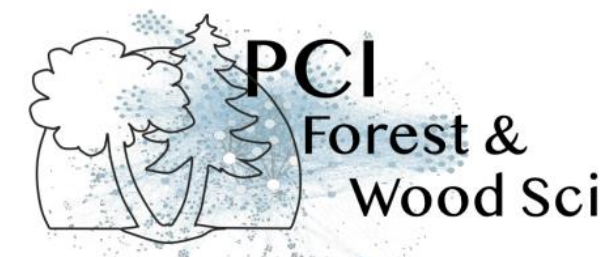

k. In table 1, there is a problem with the header of column 1;

I. "classified as epigeal with reserve type": please clarify ("type" is strange here);

\section{Instructions to format the last version of your preprint}

\section{Mandatory modifications}

1- Please make sure that:

-Data are available to readers, either in the text or through an open data repository such as Zenodo (free), Dryad or some other institutional repository. Data must be reusable, thus metadata or accompanying text must carefully describe the data.

-Details on quantitative analyses (e.g., data treatment and statistical scripts in R, bioinformatic pipeline scripts, etc.) and details concerning simulations (scripts, codes) are available to readers in the text, as appendices, or through an open data repository, such as Zenodo, Dryad or some other institutional repository. The scripts or codes must be carefully described so that they can be reused.

-Details on experimental procedures are available to readers in the text or as appendices. Include information about ethical approval for animal experimentation. Provide information about the compliance of their work with ethical standards of their national ethical committees and report the reference number of the ethical committee approval. If the study did not require ethical approval, include some sentences explaining why the approval was not needed.

-Authors have no financial conflict of interest relating to the article. The article must contain a "Conflict of interest disclosure" paragraph before the reference section containing this sentence:

"The authors of this preprint declare that they have no financial conflict of interest with the content of this article." If appropriate, this disclosure may be completed by a sentence indicating that some of the authors are PCI recommenders: "XXX is one of the PCI Forest Wood Sci recommenders."

2- Please make the following changes:

-Add the following sentence in the acknowledgements: "Version 3 of this preprint has been peerreviewed and recommended by Peer Community In Forest and Wood Sciences

(https://doi.org/10.24072/pci.forestwoodsci.100004)"

-If you use bioRxiv to post your preprint, add this latter sentence also in the "revision summary" section of the deposit form of bioRxiv.

Note that this DOI is not the DOI of your article, but the DOI of the recommendation text. The DOI of your article remains unchanged.

3- If not yet done, please send us a picture for which you own the rights that could serve as a thumbnail/illustration for your article on the web site of $\mathrm{PCl}$. It can be a figure of the article.

Optional instructions (we strongly advise you to follow them)

1 -We suggest you to remove line numbering from the preprint and put the tables and figures within the text rather than at the end of your MS.

2- Then, we strongly advise you to use the $\mathrm{PCI}$ templates (word docx template or latex template) to format your preprint in a $\mathrm{PCl}$ style. Here is the links of the templates:

https://peercommunityin.org/templates/

$\rightarrow$ For word template:

Do not hesitate to modify the template as you want (and send it back to us if you made significant improvements).

-the text to be replaced by your own text starts with XXX, eg XXXXTitle of the article.

-XXXXthe "cite_as" $\rightarrow$ Lefebvre, M., Villar, M., Boizot, N., Delile, A., Dimouro, B., Lomelech, A.-M. and Teyssier, C. (2021) Variability in seeds' physicochemical characteristics, germination and seedling growth within and between two French Populus nigra populations. arXiv, 2008.05744, ver 3 peer-reviewed and recommended by Peer community in Forest and Wood Sciences.

https://arxiv.org/abs/2008.05744 


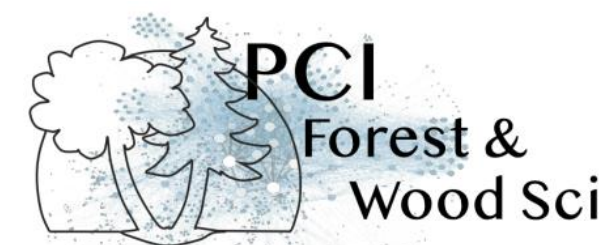

$-X X X X$ the date of deposit in the preprint server $\rightarrow$ date of the deposit of the latest version

$-X X X X$ the surnames and names of the reviewers we sent you $\rightarrow$ Tomasz A. Pawlowski, Eduardo Notivol and one anonymous reviewer

-XXXXthe_doi_we_sent_you $\rightarrow$ https://doi.org/10.24072/pci.forestwoodsci.100004

$-X X X X$ the surname and name of the recommender $\rightarrow$ Erwin Dreyer

-In the acknowledgements, add this sentence $\rightarrow$ "Version 3 of this preprint has been peer-reviewed and recommended by Peer Community In Forest and Wood Sciences

(https://doi.org/10.24072/pci.forestwoodsci.100004)"

-Please be careful to choose the badges "Open Code" and "Open Data" only if appropriate (in addition to the "Open Access" and "Open Peer-Review" badges).

$\rightarrow$ For Latex and mode org templates:

Do not hesitate to modify the template as you want (and send it back to us if you made significant improvements).

-main.tex and sample.bib should be filled.

-in main.tex, the recommender's name is "Erwin Dreyer" and the reviewers' names are Tomasz A.

Pawlowski, Eduardo Notivol and one anonymous reviewer

-In sample.bib, indicate the right version of your preprint. It is version 3

-Preambule_forestwoodsci.tex should be modified (comment lines 115, 119) to select badges.

Please be careful to choose the badges "Open Code" and "Open Data" only if appropriate (in addition to the "Open Access" and "Open Peer-Review" badges).

3- we suggest that you deposit a copy of your MS in zenodo.org and ask for its inclusion in the $\mathrm{PCl}$ community ("Communities" section in the deposit form). Indicate the current doi of your MS, if it already has one, in the "doi" section.

We hope this is clear. Do not hesitate to ask for any help if needed.

Once you have made these modifications, you should upload the new version of the article on the preprint server. Please tell us when you have done so. Thanks.

\section{Revision round \#1}

2020-11-19

\section{Author's Reply}

Download author's reply (PDF file)

\section{Decision round \#1}

\section{Dear authors}

This is a very nice and clearly presented preprint dealing with a comparison of seed properties in two contrasting french populations of Populus nigra $L$, a riparian tree species submitted to severe threads due to mismanagement of riparian excosystems and to genetic hybridization with other poplars. Important efforts are currently made for the conservation of this species present across Europe but restricted mostly to river banks. Your work concentrates on seed properties of two quite different french populations of black poplar, one along the Loire river, and another one along the Drome river in south eastern France.

Our knowledge about seed properties of this species needed be completed, and a comparison of intra and interpopulation variability in seed properties is really welcome. The preprint describes many 


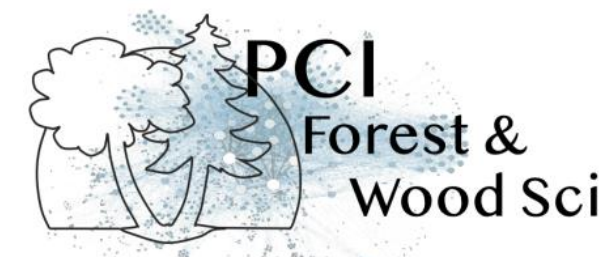

properties of these small and recalcitrant seeds, with the ambtion to relate those properties with propagation of black poplar under natural conditions. The manuscript was submitted to two experts about seed physiology, who found the study very interesting. One of the reviewers expressed nevertheless some concerns about some aspects of the preprint with which I fully concur. I did also a careful reading of the preprint myself, and made a number of suggestions and comments directly on the atttached version of the preprint (see below).

I therefore reached the conclusion that the preprint could be recommended by the $\mathrm{PCI}$ Forest \& Wood Sciences under the condition of a careful revision along the points raised by the external reviewers and by myself.

1. The manuscript is in general very well written and clearly presented. However, a number of small typos need be corrected, and some sentences require rewriting (see comments in the attached version).

2. The manuscript also refers to supplementary material. My recommendation would be either to integrate the most important material as an annex, or to add it to the data set available on the INRAE data portal.

3. The approach used remains rather descriptive (albeit very clear). I wonder whether it would not be possible to provide some more explicit research questions or even testable hypotheses. The structure of the discussion (which is very clear), defines some important focus points that should already be identified at the end of the introduction under the form of questions or hypotheses. This would be of real help to the reader and for structuring the presentation of the results.

4. The discussion refers in places to results (which should be as much as possible avoided) and sometimes provides sentences that belong rather to the introduction (I identified such sentences in the attached copy of the preprint).

5. Data display should be standardised to follow the general rule of showing three significant figures (implying a 1 permil accuracy).

6. Thank you very much for providing access to the data sets. I would recommend to cite the data set with its $\mathrm{DOI}$ in the reference list.

7. I would also recommend to provide the DOls of the papers cited in the reference list when they are available. This would enable readers to easily retrive the cited material.

8. I found also that some references were missing.

In addition to these presentation details, I have a few more general points that deserve some attention: 1 . The main aim is to document the ability of black poplar to propagate by seeds in these particular environments where the establishment of seedlings depends so strongly of the level of water in the river. I wondered whether, similarly to other poplars (for instance aspen, or P. euphratica) black poplar is able to propagate by root suckers (vegetative propagation) or whether it depends solely on seed dissemination and germination. The second question would be related to the ability of deriving rather general conclusions jabout dissemination and stand regeneration from seeds, just by describing seed properties and germination under very peculiar conditions (in Petri dishes). I had the feeling of a gap between the two scales (seed properties and in situ regeneration). However, I found the discussion quite clear in this respect with no overinterpretation of the data. This feeling is probably due to the lack of explicit description of the experimental framework in the intro (see also above). 2. Two populations is rather a small number (albeit already a lot of work) to provide clear conclusions about the correlations between seed properties and climate. Are there other data published that could help better discuss this issue from a broader range of populations?

I therefore warmly recommend to revise the preprint, taking into account the insightful comments of the referees. I believe this should be a rather easy task, and should ultimately lead to a very nice preprint I would be pleased to recommend. While submitting this revision, please prepare a point by point reply to our comments.

I am looking forward to the pleasure of considering the revised version for a recommendation.

With best regards and my apologies for the time it took to handle this preprint. 


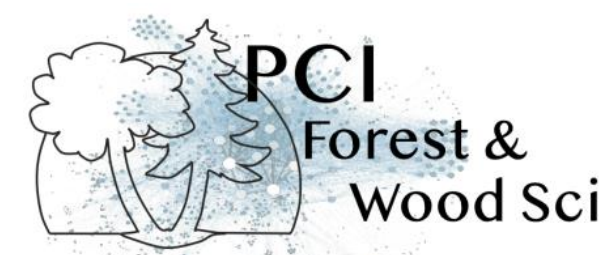

Erwin Dreyer.

\section{Additional requirements of the managing board:}

As indicated in the 'How does it work?' section and in the code of conduct, please make sure that: -Data are available to readers, either in the text or through an open data repository such as Zenodo (free), Dryad or some other institutional repository. Data must be reusable, thus metadata or accompanying text must carefully describe the data.

-Details on quantitative analyses (e.g., data treatment and statistical scripts in R, bioinformatic pipeline scripts, etc.) and details concerning simulations (scripts, codes) are available to readers in the text, as appendices, or through an open data repository, such as Zenodo, Dryad or some other institutional repository. The scripts or codes must be carefully described so that they can be reused. -Details on experimental procedures are available to readers in the text or as appendices. -Authors have no financial conflict of interest relating to the article. The article must contain a "Conflict of interest disclosure" paragraph before the reference section containing this sentence: "The authors of this preprint declare that they have no financial conflict of interest with the content of this article." If appropriate, this disclosure may be completed by a sentence indicating that some of the authors are $\mathrm{PCl}$ recommenders: "XXX is one of the $\mathrm{PCI} X X X$ recommenders."

\section{Download recommender's annotations (PDF) \\ Preprint DOI: https://data.inrae.fr/privateurl.xhtml?token=11a50eec-e00a-43a7-8fe5- dd 8 a34edffbb}

\section{Reviewed by anonymous reviewer, 2020-11-16 12:18}

This manuscript addresses an important issue for understanding of the physiology, ecology and regeneration requirements of black poplar (Populus nigra). I agree with the Authors that our knowledge on the chemical constitution of seeds of tree species is scare, and more information about it will allow us to improve our understanding of tree adaptation to changing environment. Additionally, such research should be done at the first place on species threatened with extinction such as black poplar or economically significant. Therefore, I think that this article could be interesting for broad scientific community, particularly scientists gathered on the topic on seeds biochemistry as well as plant ecology. The presented manuscript is consistent and well written. The statements are clear to me. The results are presented in a transparent and clear manner. The only minor drawback of the research is a sparse climatic data which is limited to average temperature only. It seems to me that work would gain a lot if this part was expanded. Nevertheless, in my opinion the presented manuscript is very interesting could be published in PCI Forest Wood Sci.

\section{Reviewed by Tomasz A. Pawlowski, 2020-11-06 11:49}

Dear Professor Erwin Dreyer,

I have completed evaluation of proposed preprint.

Yours sincerely,

Tomasz Pawłowski

Revision of the manuscript entitled "Variability in seeds' physicochemical characteristics, germination and seedling growth within and between two French Populus nigra populations" for PCI Forest \& Wood Sci.

\section{Comments to the Authors}

The authors report about analysis of the biochemical composition of Populus nigra seeds in two contrasting environments in France. Significant between population and individual trees differences were detected.

General comment: the paper is well written and the topic has been studied in depth by the authors. The achieved data is important in the prediction of the regeneration requirements of black poplar. 


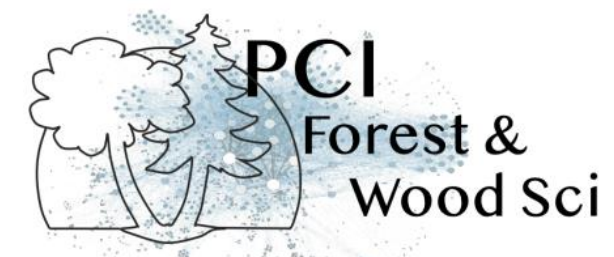

Remarks

Materials and Methods

Fresh weigh, dry weight, and water content.

The methods of fw and dw determination were not clearly described. Was determined for years 2017 -2019 and two populations only dw, and for 2019 and Loire only fw? That should be explained.

Results

Table 2. What I see from it is a lack of some sample fw and dw determination. What was a reason of that? Moreover, family and population effects were showed, but the time factor could be the interesting approach in these investigations. Why this was not investigated during the study despite seed collections during three years (as for dw determination)? These information could make this work more interesting.

Fig. 2. Show the arrows properly the investigated bands?

Discussion

Structural and biochemical characterisation of the seeds.

The Populus nigra seds were classified as recalcitrant as well as intermediate, so that should be taken into account during discussion. ...the Populus nigra seeds we examined had far higher contents of storage proteins (ca. $60 \%$ of total proteins; Fig. 2), than of structural and enzymatic proteins.

How was this proven because results did not showed that?

Loire seeds had higher dw than Drôme seeds, as the temperature is warmer () at the Drôme site than the Loire site.

It means that colder temperature cause the dw increase. How you can explain that?

Consequences of seed size for germination and growth of juvenile seedlings

Did you observe the correlation between family (individual tree) seed size and seedlings growth?

Seedling emergence is sometimes better parameter of seed quality than germination rate, however for light seeds as are black poplar the influence of photosynthesis can be main factor of the differences in seedlings growth.

\section{Reviewed by Eduardo Notivol, 2020-11-26 11:32}

Through biochemical composition of seeds the draft studies the variability of families (2x10) in two contrasting rivers (Drôme and Loire), looking for relationships between composition and germination and intra- and inter-population variability. It seems to be the first reference on biochemical composition of species' seeds, and this is worthy to mention.

Main findings are

Family variation (important for adaptation) In some traits population differentiation (lipids and $\mathrm{s}$. sugars) but not in others (proteins). One population always bigger than other (3 years) No differences on germination. One population longer juvenile roots No critical factors in selection pressure

The paper is well written and presented. Methodology is well-defined and proper and the results are clearly exposed. Discussion is rich and raises interesting ideas about the relationships between the morphological traits, biochemical composition and germination and early steps of the seedlings.

I enjoyed reading the draft and only have very light comments for improvement:

Perhaps the introduction is a little too long even though it provides a comprehensive description and justification of the study.

In Mat and Met, for biochemical procedures there is no description about the family sampling, only number of biological replicates of seeds with weight is described but not about families' information, same for carbohydrates and lipids. It lacks some reference (like in the germination description they do) "per family".

In table 2 caption describe dw and and fw in the same way than WC 


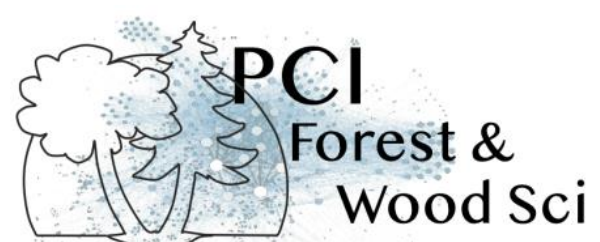

In Fig 3 Drôme ellipse is too big and comprises 3 Loire trees, if you diminish the size of it the difference between populations will be better shown. 